\title{
Determinação do teor de umidade em grãos empregando radiação micro-ondas
}

\author{
Determination of grain moisture content using microwave radiation
}

\author{
Matheus Rafael Raschen ${ }^{\mathrm{I}}$ Fernanda Bortoluzzi Lucion ${ }^{\mathrm{I}}$ Alexandre José Cichoski ${ }^{\mathrm{I}}$ \\ Cristiano Ragagnin de Menezes ${ }^{\mathrm{I}}$ Roger Wagner $^{\mathrm{I}}$ Eduardo Jacob Lopes ${ }^{\mathrm{I}}$ \\ Leila Queiroz Zepka ${ }^{\mathrm{I}}$ Juliano Smanioto Barin ${ }^{\mathrm{I}}$
}

\section{RESUMO}

A determinação de umidade em grãos é um parâmetro de qualidade importante, uma vez que o teor de água presente no alimento tem influência tanto no armazenamento quanto na comercialização do produto. Os métodos usados como referência na determinação de umidade em grãos empregam a perda por dessecação em estufa (LOD). Apesar de ser de fácil execução, esse método dispende cerca de $24 \mathrm{~h}$ de aquecimento, normalmente, em $105^{\circ} \mathrm{C}$, para obtenção do resultado. No presente estudo, é proposta a determinação da perda por dessecação assistida por radiação micro-ondas (MALOD) para amostras de grãos moídos de arroz branco, arroz integral, ervilha, feijão branco, feijão carioca, feijão preto, grão-de-bico, lentilha e milho, buscando reduzir o tempo de análise. Parâmetros como potência irradiada, massa de amostra, influência do recipiente e tempo de irradiação com micro-ondas foram avaliados e os resultados obtidos foram comparados com a LOD (sugerido pelo MAPA) e, também, por radiação infravermelha (IRLOD). Verificou-se que não houve diferença significativa do teor de umidade empregando massas de amostra entre 2 e $15 \mathrm{~g}$ por $L O D$, porém, para MALOD, ocorreram hot spots (carbonização da amostra) com massas superiores a $2 \mathrm{~g}$. Resultados similares de umidade foram encontrados entre os diferentes recipientes (pesafiltros e frascos de polipropileno), optando-se pela utilização dos frascos de polipropileno, devido à diminuição do tempo de précondicionamento. A MALOD apresentou concordância entre $94 \%$ e 102\% com o método oficial, sendo que apenas 50min foram suficientes para determinar a umidade para todos os grãos, perfazendo uma redução do tempo de análise de até 29 vezes.

Palavras-chave: umidade, micro-ondas, grãos, perda por dessecação, determinação de água.

\section{ABSTRACT}

The determination of moisture in grains is an important quality parameter, since the water content in food has an influence on both storage and commercialization of product. The methods often used for determination of moisture in grains are based on the loss on drying in an oven (LOD). Although it is easy to perform, this method spent about $24 \mathrm{~h}$ of heating, usually at $105^{\circ} \mathrm{C}$, to obtain the result. In the present study is proposed the determination of the loss on drying assisted by microwave radiation (MALOD) for samples of grounded grains of white rice, brown rice, peas, beans, bean, black beans, chick-peas, lentils and corn, in order to reduce the analysis time. Parameters such as irradiated power, sample mass, container and influence of irradiation time with microwave were evaluated and the results were compared with those obtained by LOD (suggested by MAPA) and also by infrared radiation (IRLOD). It was found that there was no significant difference in moisture content in samples between 2 and $15 \mathrm{~g}$ using LOD, but there were hot spots for MALOD (carbonization of the sample) with masses higher than $2 \mathrm{~g}$. Similar results for moisture content were found by the use of different containers (glass weighing bottle and polypropylene flasks) and the polypropylene cups were selected due to the reduction of conditioning time of flasks. The agreement of MALOD with the official method was between $94 \%$ and $102 \%$ and only 50min were enough to determine the moisture of all grains, making a reduction of analysis time by 29 times.

Key words: moisture, microwaves, grains, loss on drying, water determination.

\section{INTRODUÇÃo}

O teor de umidade é uma informação importante da composição de alimentos e está entre os parâmetros frequentemente determinados em rotina, podendo servir como um indicador da qualidade dos produtos, uma vez que apresenta influência direta no armazenamento (VALENTINI et al., 1998; AMOEDO \& MURADIAN, 2002). Os grãos, quando armazenados em determinadas condições ambientais de umidade e de temperatura, podem

IDepartamento de Tecnologia e Ciência dos Alimentos, Universidade Federal de Santa Maria (UFSM), 97105-900, Santa Maria, RS, Brasil. E-mail: juliano@ufsm.br. *Autor para correspondência 
favorecer a propagação de fungos e a produção de micotoxinas, que são prejudiciais à saúde (D'MELLO \& MACDONALD, 1997; ATALLA et al., 2003; MILLER, 2005). Além disso, o teor de água interfere diretamente no valor econômico dos produtos, pois os grãos são comercializados, normalmente, por peso (RAY et al., 1992). Os métodos para determinação da umidade em grãos consistem, normalmente, na retirada de água dos alimentos por aquecimento, em que o teor de umidade pode ser calculado através da diferença de peso inicial e final das amostras (THIEX \& RICHARDSON, 2003; MENDONÇA et al., 2007; VUATAZ et al., 2010). Segundo BRASIL (2009), o método oficial de determinação de umidade em grãos emprega a perda por dessecação em estufa (LOD, do inglês loss on drying), empregando $105 \pm 3^{\circ} \mathrm{C}$ durante 24h. Esse método possui a vantagem de ser de simples execução e permitir o processamento de um grande número de amostras, tendo ampla utilização em diversos laboratórios. Contudo, a LOD é um procedimento moroso e inadequado para a rotina laboratorial ou para indústrias que necessitem de resultados rápidos, podendo levar horas ou até mesmo dias para a obtenção de resultados (TRABELSI \& NELSON, 2007; ILELEJI, K.E. et al., 2010).

Assim, a perda por dessecação assistida por radiação micro-ondas (MALOD, do inglês microwave-assisted loss on drying) surge como uma alternativa para substituição do método convencional, pois permite a determinação da umidade de maneira mais rápida (KING et al., 1992; VALENTINI et al., 1998). BIZZI et al. (2011) adaptaram um forno de micro-ondas doméstico para a MALOD de sacarídeos, obtendo resultados concordantes com o método oficial, com redução do tempo de análise de até 15 vezes. Neste trabalho, é proposto o uso da MALOD para amostras de diferentes grãos, buscando reduzir o tempo de análise. Parâmetros como potência irradiada, massa de amostra, tipo de recipiente e tempo de irradiação com micro-ondas foram avaliados. Os resultados foram comparados com aqueles obtidos por LOD e, também, pela perda por dessecação assistida por radiação infravermelha (IRLOD, do inglês infrared loss on drying).

\section{MATERIAL E MÉTODOS}

\section{Amostras}

As amostras de grãos (arroz branco, arroz integral, ervilha, feijão branco, feijão carioca, feijão preto, grão-de-bico, lentilha e milho) foram adquiridas no comércio local. Os grãos foram amostrados por quarteamento e cominuídos até tamanho menor que
$315 \mu \mathrm{m}$, sendo posteriormente armazenados em potes herméticos em temperatura ambiente.

Instrumentação

A moagem dos grãos foi realizada em um moinho de hélices (modelo MA-630, Marconi, Brasil) com 500W de potência. A tamisação foi efetuada em peneiras com abertura de malha de $800 \mu \mathrm{m}$ e $315 \mu \mathrm{m}$, empregando agitador (modelo 3528, Bertel, Brasil) com intensidade de vibração seis durante um minuto para cada matriz. Entretanto, somente grânulos de tamanhos inferiores a $315 \mu \mathrm{m}$ foram utilizados para a determinação do teor de umidade.

A MALOD foi realizada em um forno de micro-ondas doméstico (modelo BK035642Y240D, 31L, 2450MHz, Philco, Brasil) com 900W de potência nominal. Tendo em vista que a quantidade de amostra utilizada não permite a absorção de toda a radiação gerada pelo magnetron (gerador de ondas), uma mangueira de polietileno ( $1 \mathrm{~m}$ de comprimento e $5 \mathrm{~mm}$ de diâmetro) foi adaptada no interior do forno com um fluxo constante $\left(700 \mathrm{~mL} \mathrm{~min}^{-1}\right)$, com a função de absorver essa radiação excessiva, conforme recomendado por BIZZI etal.(2011). Como recipientes, foram empregados pesa-filtros de vidro borossilicato de $25 \mathrm{~mm}$ de diâmetro interno e volume de $30 \mathrm{~mL}$, além de frascos descartáveis para café de polipropileno $(50 \mathrm{ml})$ cobertos por folhas de papel alumínio $(7,5 \mathrm{~cm}$ $\mathrm{x} 7,5 \mathrm{~cm}$ ) somente após a etapa de aquecimento. Um detector de vazamento de microondas foi utilizado periodicamente durante os experimentos para verificar uma eventual perda de radiação pelos orifícios feitos no aparelho para passagem das mangueiras (modelo LT-2D, 2450MHz, o limite máximo de $5 \mathrm{~mW} \mathrm{~cm}^{-2}$, Milestone SRL, Sorisole, Itália). A determinação da LOD foi efetuada em estufa (modelo A5SEAF, DeLeo, Brasil). Para a pesagem, foi utilizada balança analítica (modelo Mark 250 A, máx 250g, 0,1mg de resolução, BEL, Brasil). A IRLOD foi feita em balança com irradiação no infravermelho (modelo MA150, 1mg de resolução, Sartorius, Alemanha), empregando pratos de alumínio descartáveis.

Determinação da potência real e distribuição da radiação micro-ondas no interior da cavidade do forno Para determinação da potência real no interior da cavidade do forno de micro-ondas, foi colocado um béquer de vidro contendo $1000 \mathrm{~g}$ de água destilada no forno e a temperatura foi medida através de termômetro de mercúrio antes e após a irradiação (120s, potência máxima). Já para a avaliação da distribuição de radiação, foram posicionados simetricamente nove frascos contendo $15 \mathrm{~g}$ de água destilada durante 
60s na potência máxima e a temperatura também foi medida antes e após a irradiação com micro-ondas. A determinação da potência (em Watts) foi realizada empregando a equação $\mathrm{P}=\mathrm{cm} \Delta \mathrm{T} / \mathrm{t}$, em que c é a capacidade calorífica específica da água $\left(4,184 \mathrm{~J} \mathrm{~g}^{-1}\right.$ $\left.\mathrm{K}^{-1}\right)$, m é a massa da amostra $(\mathrm{g}), \Delta \mathrm{T}$ é a variação de temperatura e t é o tempo de irradiação (s).

Determinação da perda por dessecação

A determinação da LOD foi efetuada de acordo com o procedimento recomendado pelo Ministério da Agricultura, Pecuária e Abastecimento (BRASIL, 2009). Diferentes massas de amostra (2, 5, 10 e $15 \mathrm{~g}$ ) foram pesadas em pesa-filtros de vidro précondicionados durante $30 \mathrm{~min}$ a $105^{\circ} \mathrm{C}$ e inseridas na estufa a $105^{\circ} \mathrm{C}$ por um período de $24 \mathrm{~h}$. Após a secagem, os recipientes foram mantidos em dessecador até atingirem a temperatura ambiente. Os valores da perda por dessecação foram estabelecidos pela diferença entre a massa da amostra antes e após o aquecimento. Para MALOD, cerca de $2 \mathrm{~g}$ de cada amostra foram pesadas e os recipientes foram dispostos nas regiões estabelecidas conforme o mapeamento citado anteriormente. O tempo necessário para MALOD foi avaliado no período de $50 \mathrm{~min}$, com incrementos de 10min. Para LOD e MALOD, também foi avaliada a possibilidade de substituição dos pesa-filtros de vidro por copos de polipropileno descartáveis, com vistas à redução do tempo empregado para lavagem e preparação dos recipientes (pré-condicionamento). Para tanto, foram utilizados $2 \mathrm{~g}$ de amostra de arroz branco, ervilha, feijão carioca e lentilha em ambos os recipientes (vidro e polipropileno, em triplicatas). Para os frascos de polipropileno, uma bandeja plástica de polipropileno (diâmetro de $15 \mathrm{~cm}$ ) foi empregada para acondicionar os frascos, sendo posicionada a uma altura de $0,8 \mathrm{~cm}$ do prato giratório de vidro.

Para IRLOD, $2 \mathrm{~g}$ de amostra foram pesadas diretamente no equipamento e diferentes temperaturas foram utilizadas $\left(105^{\circ} \mathrm{C}, 120^{\circ} \mathrm{C}, 150^{\circ} \mathrm{C}\right.$ e $\left.180^{\circ} \mathrm{C}\right)$. O final da análise foi determinado após estabilização da perda por dessecação das amostras, considerando uma variação menor que $0,001 \mathrm{~g}$ em um período de, pelo menos, $2 \mathrm{~min}$. As médias foram comparadas pelo teste de Tukey em nível de 5\% de probabilidade de erro.

\section{RESULTADOS E DISCUSSÃO}

Determinação da potência real e distribuição da radiação no interior da cavidade do forno de micro-ondas

A potência real encontrada em potência máxima, através dos testes realizados, foi de $697 \mathrm{~W}$, o que corresponde a $77,4 \%$ da potência nominal. O mapeamento da radiação no interior do forno demonstrou a heterogeneidade da incidência das ondas, ressaltando a importância de disponibilizar de forma adequada os copos na cavidade do forno de micro-ondas. Na faixa entre 0 e $5 \mathrm{~cm}$ de distância, a partir do centro do prato giratório, a potência obtida foi $43,9 \mathrm{~W}$, já entre 5 e $10 \mathrm{~cm}$ obteve-se $41,8 \mathrm{~W}$. A posição selecionada para a realização dos experimentos ficou na faixa entre 10 e $15 \mathrm{~cm}$ do centro do prato giratório, pois foi a região de maior incidência de radiação micro-ondas $(45,0 \mathrm{~W})$.

Influência da massa de amostra e do recipiente para LOD e MALOD

Diferentes massas de amostra de feijão branco $(2,5,10$ e $15 \mathrm{~g})$ foram avaliadas para LOD e nenhuma diferença significativa do teor de umidade foi observada. Dessa forma, $2 \mathrm{~g}$ de amostra foram selecionados para os experimentos subsequentes. Já no teste realizado no forno de micro-ondas. para amostras de feijão branco e milho durante $50 \mathrm{~min}$, observou-se a formação de hot spots com $5 \mathrm{~g}$ de amostra e até mesmo carbonização com massas de 8 , 10 e $15 \mathrm{~g}$, o que poderia levar a erros sistemáticos, devido à perda de massa nessas condições. Dessa forma, a massa escolhida para a realização dos experimentos também foi de $2 \mathrm{~g}$. A mesma massa também foi mantida para os experimentos de IRLOD.

A comparação entre os diferentes recipientes provou que não houve diferença considerável entre os resultados. Para pesa-filtros de vidro e frascos de polipropileno, foi observada uma média de umidade de $12,50 \% \pm 0,05$ e $12,40 \% \pm 0,06$ para arroz branco, $12,97 \% \pm 0,05$ e $12,87 \% \pm 0,06$ para ervilha, $13,31 \% \pm 0,07$ e $13,22 \% \pm 0,05$ para feijão carioca e $13,56 \% \pm 0,08$ e $13,67 \% \pm 0,16$ para lentilha, respectivamente, e nenhuma diferença significativa foi encontrada (teste $\mathrm{t}$ de Student, $\mathrm{P}<0,05$ ). Dessa forma, optou-se pela substituição dos pesa-filtros de vidro pelos frascos de polipropileno na determinação da perda por dessecação, pois torna desnecessária a etapa inicial do processo (pré-condicionamento), que demanda tempo excessivo para a eliminação de qualquer umidade presente no recipiente. Isso se deve à natureza hidrofóbica do recipiente plástico, que evita a adsorção de água e permite a introdução direta da amostra no frasco. Ao contrário dos pesa-filtros, os frascos de polipropileno não necessitam ser limpos, uma vez que são descartáveis, acelerando ainda mais o procedimento. Além disso, por possuírem menor massa que os pesa-filtros, ajudam a minimizar os erros associados ao processo de pesagem, uma vez 
que o procedimento está fundamentado na diferença de peso antes e após o aquecimento das amostras.

Determinação do tempo de secagem por MALOD

O perfil de perda por dessecação (Figura 1) foi realizado com o objetivo de determinar qual o tempo necessário para as amostras atingirem o valor máximo de perda de massa. Nos primeiros 10min de aquecimento, as amostras apresentaram resultados superiores a $59 \%$ do valor total de umidade presente, mostrando que grande parte da água é perdida nessa etapa. A amostra de feijão preto teve a secagem finalizada com apenas $30 \mathrm{~min}$, entretanto, para as amostras de grão-de-bico, feijão carioca, milho, feijão branco, lentilha e ervilha, foi observado que o tempo necessário para remover toda umidade ocorreu com 40min. O mesmo tempo de irradiação com micro-ondas foi observado por Da LUZ et al. (1998) para amostras de sementes de arroz moídas. Entretanto, para tornar o procedimento mais simples e assegurar que todas as amostras recebessem quantidade suficiente de ondas para completar a perda por dessecação, optou-se por utilizar o tempo de 50min de irradiação para todas as amostras nos experimentos subsequentes.

Determinação da perda por dessecação por LOD, MALOD e IRLOD

Os dados da tabela 1 demonstram os resultados da perda por dessecação de diferentes grãos, empregando LOD, IRLOD e MALOD. Para todos os grãos estudados, os valores encontrados para MALOD apresentaram concordância entre 94 e 102\% em relação ao método oficial (LOD). Uma baixa variabilidade entre as replicatas foi observada (desvio-padrão relativo inferior a 2,3\%), demonstrando que a MALOD apresenta precisão semelhante à LOD.

As diferentes temperaturas empregadas em IRLOD apresentaram resultados distintos entre

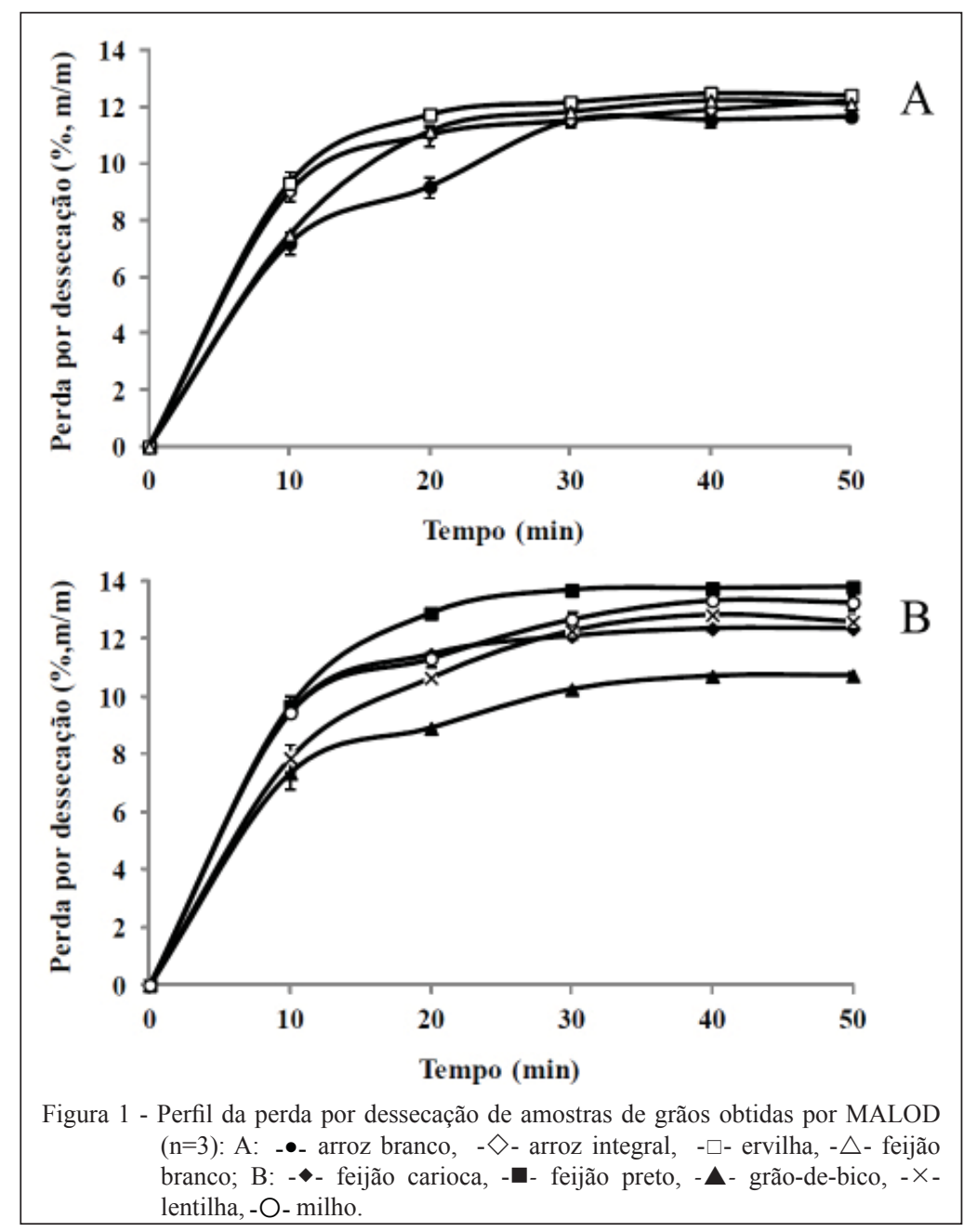

Ciência Rural, v.44, n.5, mai, 2014. 
Tabela 1 - Resultados obtidos para a determinação da umidade por perda por dessecação (\%, m/m) de grãos utilizando LOD, IRLOD e $\operatorname{MALOD}(\mathrm{n}=4)$.

\begin{tabular}{|c|c|c|c|c|c|c|}
\hline \multirow{2}{*}{ Amostras } & \multirow{2}{*}{ LOD* } & \multicolumn{5}{|c|}{ 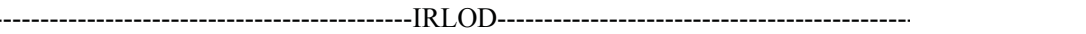 } \\
\hline & & $105^{\circ} \mathrm{C}$ & $120^{\circ} \mathrm{C}$ & $150^{\circ} \mathrm{C}$ & $180^{\circ} \mathrm{C}$ & \\
\hline Arroz branco & $11,96 \pm 0,10^{\mathrm{b}}$ & $10,32 \pm 0,17^{\mathrm{d}}$ & $10,88 \pm 0,15^{\mathrm{c}}$ & $12,07 \pm 0,02^{\mathrm{b}}$ & $12,98 \pm 0,01^{\mathrm{a}}$ & $11,66 \pm 0,18^{b}$ \\
\hline Arroz integral & $12,19 \pm 0,05^{\mathrm{a}}$ & $10,56 \pm 0,03^{\mathrm{b}}$ & $10,81 \pm 0,08^{\mathrm{b}}$ & $12,23 \pm 0,13^{\mathrm{a}}$ & $12,31 \pm 0,10^{\mathrm{a}}$ & $12,21 \pm 0,22^{\mathrm{a}}$ \\
\hline Ervilha & $12,19 \pm 0,14^{\mathrm{c}}$ & $11,88 \pm 0,07^{\mathrm{cd}}$ & $12,01 \pm 0,17^{\mathrm{b}}$ & $12,70 \pm 0,04^{\mathrm{b}}$ & $13,23 \pm 0,04^{\mathrm{a}}$ & $12,41 \pm 0,09^{\mathrm{bc}}$ \\
\hline Feijão branco & $12,31 \pm 0,09^{\mathrm{c}}$ & $11,63 \pm 0,17^{\mathrm{d}}$ & $12,88 \pm 0,03^{b}$ & $13,00 \pm 0,27^{\mathrm{ab}}$ & $13,45 \pm 0,05^{\mathrm{a}}$ & $12,11 \pm 0,12^{\mathrm{c}}$ \\
\hline Feijão carioca & $12,23 \pm 0,29^{\mathrm{ab}}$ & $11,63 \pm 0,17^{\mathrm{b}}$ & $12,01 \pm 0,71^{\mathrm{ab}}$ & $12,63 \pm 0,01^{\mathrm{ab}}$ & $13,59 \pm 0,41^{\mathrm{a}}$ & $12,36 \pm 0,07^{\mathrm{ab}}$ \\
\hline Feijão preto & $14,25 \pm 0,17^{\mathrm{ab}}$ & $12,95 \pm 0,17^{\mathrm{d}}$ & $13,49 \pm 0,01^{\mathrm{cd}}$ & $13,53 \pm 0,47^{\mathrm{cd}}$ & $14,72 \pm 0,11^{\mathrm{a}}$ & $13,79 \pm 0,21^{\mathrm{bc}}$ \\
\hline Grão-de-bico & $11,27 \pm 0,16^{\mathrm{b}}$ & $11,10 \pm 0,13^{\mathrm{bc}}$ & $11,19 \pm 0,03^{b}$ & $11,36 \pm 0,16^{\mathrm{b}}$ & $12,18 \pm 0,03^{\mathrm{a}}$ & $10,73 \pm 0,18^{\mathrm{c}}$ \\
\hline Lentilha & $12,76 \pm 0,12^{\mathrm{bc}}$ & $12,62 \pm 0,04^{\mathrm{bc}}$ & $12,83 \pm 0,17^{\mathrm{bc}}$ & $13,37 \pm 0,40^{\mathrm{b}}$ & $14,34 \pm 0,04^{\mathrm{a}}$ & $12,60 \pm 0,28^{\mathrm{bc}}$ \\
\hline Milho & $13,63 \pm 0,15^{\mathrm{ab}}$ & $11,47 \pm 0,11^{\mathrm{d}}$ & $11,86 \pm 0,28^{\mathrm{d}}$ & $12,86 \pm 0,09^{\mathrm{c}}$ & $13,85 \pm 0,24^{\mathrm{a}}$ & $13,26 \pm 0,24^{\mathrm{bc}}$ \\
\hline
\end{tabular}

Médias não seguidas pela mesma letra em cada coluna diferem entre si pelo teste de Tukey em nível de 5\% de probabilidade de erro. $* 105^{\circ} \mathrm{C}, 24 \mathrm{~h}$ (MAPA)

** 50min.

os grãos estudados, demonstrando que não houve uma temperatura padrão em que todas as amostras pudessem apresentar resultados concordantes com a LOD. Com $105^{\circ} \mathrm{C}$, todas as amostras apresentaram resultados inferiores à LOD, com exceção de grãode-bico e lentilha. Optou-se, então, pela utilização de uma temperatura superior $\left(120^{\circ} \mathrm{C}\right)$, em que as amostras de ervilha, feijão branco, feijão carioca e grão-de-bico apresentaram resultados favoráveis. Entretanto, em $120^{\circ} \mathrm{C}$ as amostras de arroz branco, arroz integral, feijão preto e milho não alcançaram resultados comparáveis ao método de referência e, dessa maneira, optou-se, então, pela utilização de $150^{\circ} \mathrm{C}$. Para as amostras de arroz branco e arroz integral, valores satisfatórios foram obtidos nessa temperatura, porém, para feijão preto e milho, foi necessária a utilização de $180^{\circ} \mathrm{C}$. Os resultados discrepantes obtidos para IRLOD estão de acordo com o observado por Mendonça e colaboradores para amostras de café verde, em que resultados superiores foram obtidos com o emprego da LOD (MENDONÇA et al., 2007).

Como pode ser observado na tabela 1 , algumas amostras submetidas à temperatura de $180^{\circ} \mathrm{C}$ apresentaram valores superiores ao desejado. Durante a secagem nessas condições, notaram-se modificações na coloração dos grãos (escurecimento), indicando a degradação da amostra. Essa degradação possibilita a ocorrência de reações indesejáveis, que podem levar à formação de substâncias voláteis e que levam a resultados superestimados e errôneos. Portanto, para o uso da IRLOD, torna-se necessário estabelecer a melhor temperatura para cada tipo de amostra, não sendo possível a utilização de um procedimento único para diferentes amostras, como pode ser observado para LOD e MALOD.

Comparação da perda por dessecação empregando LOD, MALOD e IRLOD

Os dados da tabela 2 demonstram os parâmetros avaliados para os diferentes métodos estudados. A MALOD possui vantagem em diversos aspectos se comparada aos métodos LOD e IRLOD. Foi possível determinar a umidade por MALOD empregando até 18 frascos durante o período de $50 \mathrm{~min}$, frente a $1440 \mathrm{~min}(24 \mathrm{~h})$ consumidos pela LOD, permitindo uma redução de tempo no procedimento de cerca de 29 vezes. A IRLOD destaca-se por empregar um equipamento automatizado e de fácil operação, porém, necessita de estudos prévios para determinar o tempo e as temperaturas necessárias para a secagem. A principal desvantagem desse método é a realização do procedimento utilizando apenas uma amostra por vez, o que aumenta significativamente o tempo de análise.

Tabela 2 - Parâmetros de mérito avaliados relativos aos procedimentos de determinação da perda por dessecação.

\begin{tabular}{llll}
\hline Parâmetro & LOD & MALOD & IRLOD \\
\hline $\begin{array}{l}\text { Tempo (min) } \\
\begin{array}{l}\text { Frequência de análise } \\
\left(\mathrm{n}^{\circ} \text { de amostras } \mathrm{h}^{-1}\right)\end{array}\end{array}$ & 1440 & $40-50$ & $6-12$ \\
$\begin{array}{l}\text { Custo } \\
\text { Gasto de energia }\end{array}$ & $\begin{array}{l}\text { baixo-médio } \\
\text { alto }\end{array}$ & $\begin{array}{l}\text { Baixo } \\
\text { Baixo }\end{array}$ & $\begin{array}{l}\text { alto } \\
\text { baixo }\end{array}$ \\
\hline
\end{tabular}

*Considerando 72 amostras por ciclo. 
Com relação ao parâmetro de gasto de energia, o método oficial possui um elevado consumo, pois demanda tempo excessivo de utilização (24h). O contrário ocorre com MALOD e IRLOD que, por necessitarem de menor tempo, possuem um gasto de energia inferior. Referente aos custos dos equipamentos, a LOD possui valor de aquisição e manutenção considerado de baixo a médio, ao contrário de MALOD, que utiliza um aparelho doméstico comumente encontrado no comércio local. Já para IRLOD os custos de obtenção do aparelho são elevados, tornando o procedimento inviável para muitos laboratórios analíticos.

\section{CONCLUSÃO}

O método de perda por dessecação assistida por radiação micro-ondas (MALOD) pode ser utilizado como uma alternativa de substituição ao método convencional (LOD), reduzindo o tempo de secagem dos grãos em até 29 vezes. O equipamento pode ser adaptado facilmente em laboratório a baixo custo. Além disso, a substituição dos pesa-filtros de vidro por frascos de polipropileno acelerou o processo de secagem, eliminando a etapa de précondicionamento dos recipientes de vidro, tornando o procedimento ainda mais atraente para a rotina laboratorial.

\section{REFERÊNCIAS}

AMOEDO, L.H.G.; MURADIAN, L.B.A. Comparação de metodologias para a determinação de umidade em geléia real. Química Nova, v.25, p.676-679, 2002. Disponível em: <http:// www.scielo.br/pdf/qn/v25n4/10544.pdf $>$. Acesso em: $12 \mathrm{jul}$. 2011. doi: 10.1590/S0100-40422002000400024.

ATALLA, M.M. et al. Mycotoxin production in wheat grains by different Aspergilli in relation to different relative humidities and storage periods. Nahrung/Food, v.47, p.6-10, 2003. Disponível em: <http://onlinelibrary.wiley.com/doi/10.1002/food.200390017/ pdf>. Acesso em: 25 jul. 2011. doi: 10.1002/food.200390017.

BIZZI, C.A. et al. A fast microwave-assisted procedure for loss on drying determination in saccharides. Journal Brazilian Chemistry Society, v.22, p.377-381, 2011. Disponível em: $<$ http://www.scielo.br/scielo.php?script=sci_arttext\&pid $=$ S010350532011000200026>. Acesso em: 17 jul. 2011. doi: 10.1590/S0103-50532011000200026.

BRASIL. Ministério da Agricultura Pecuária e do Abastecimento. Regras para análise de sementes. Brasília, DF: SNDA/DNDV/ CLAV, 2009. Cap.7, p.307-323. Disponível em: <http://www. bs.cca.ufsc.br/publicacoes/regras\%20analise \%20sementes.pdf $>$. Acesso em: 12 set. 2012.
DA LUZ, C. et al. Determinação do teor de água de sementes de arroz por secagem com microondas. Revista Brasileira de Sementes, v.20, n.1, p.70-74, 1998. Disponível em: <http://www. abrates.org.br/revista/artigos/1998/v20n1/artigo13.pdf $>$. Acesso em: 12 set. 2012 .

D’MELLO, J.P.F.; MACDONALD, A.M.C. Mycotoxins. Animal Feed Science Technology, v.69, p.155-166, 1997. Disponível em: <http://www.sciencedirect.com/science/article/pii/ S0377840197816306>. Acesso em: 25 jul. 2011. doi: 10.1016/ S0377-8401(97)81630-6.

ILELEJI, K.E. et al. Comparison of standard moisture loss-ondrying methods for the determination of moisture content of corn distillers dried grains with solubles. Journal of AOAC International, v.93, n.3, p.825-832, 2010. Disponível em: $<$ http://aoac.publisher.ingentaconnect.com/content/aoac/ jaoac/2010/00000093/00000003/art00013>. Acesso em: 12 set. 2012 .

KING, R.J. et al. Microwave moisture measurement of grains. IEEE Transactions on Instrumentation and Measurement, v.41, n.1, p.111-115, 1992. Disponível em: <http://ieeexplore.ieee. $\mathrm{org} / \mathrm{stamp} /$ stamp.jsp?tp=\&arnumber=126643>. Acesso em: 17 jul. 2011. doi: $10.1109 / 19.126643$.

MENDONÇA, J.C.F. et al. A comparative evaluation of methodologies for water content determination in green coffee. LWT - Food Science and Technology, v.40, n.7, p.1300-1303, 2007. Disponível em: <http://www.sciencedirect.com/science/ article/pii/S0023643806002337>. Acesso em: 12 set. 2012. doi: 10.1016/j.lwt.2006.08.013.

MILLER, J.D. Fungi and mycotoxins in grain: implications for stored product research. Journal of Stored Products Research, v.31, p.1-16, 1995. Disponível em: <http://www.sciencedirect. com/science/article/pii/0022474X9400039V>. Acesso em: 25 jul. 2011. doi: 10.1016/0022-474X(94)00039-V.

THIEX, N.; RICHARDSON C.R. Challenges in measuring moisture content of feeds. Journal of Animal Science, v.81, p.3255-3266, 2003. Disponível em: <http://jas.fass.org/ content/81/12/3255.full.pdf>. Acesso em: 20 jul. 2011.

TRABELSI, S; NELSON, O.S. Unified microwave moisture sensing technique for grain and seed. Measurement Science Technology, v.18, p.997-1003, 2007. Disponível em: <http:// iopscience.iop.org/0957-0233/18/4/006/>. Acesso em: 17 jul. 2011. doi: $10.1088 / 0957-0233 / 18 / 4 / 006$.

VALENTINI, S.R. et al. Determinação do teor de umidade de milho utilizando aparelho de microondas. Ciência e Tecnologia de Alimentos, v.18, n.2, p.237-240, 1998. Disponível em: <http:// www.scielo.br/scielo.php?pid $=$ S010120611998000200017\&scri pt=sci_arttext. $>$. Acesso em: 13 jul. 2011. doi: 10.1590/S0101$206119 \overline{9} 98000200017$.

VUATAZ G. et al. TG-DTA approach for designing reference methods for moisture content determination in food powders. Food Chemistry, v.122, n.2, p.436-442, 1998. Disponível em: <http:// www.sciencedirect.com/science/article/pii/S0308814609007675>. Acesso em: 12 set. 2012. doi: 10.1016/j.foodchem.2009.05.066. 